Witold Miecznikowski ${ }^{1}$, Paweł Kiczmer ${ }^{2}$ (D) Alicja Prawdzic-Seńkowska² ${ }^{\circledR}$, Karolina Cygan ${ }^{3}$, Elżbieta Świętochowska²

${ }^{1}$ Department of Orthopedics, Medical University of Silesia, Trauma Center, Sosnowiec, Poland

2Department of Medical and Molecular Biology, School of Medicine with the Division of Dentistry, Medical University of Silesia in Zabrze,

Katowice, Poland.

${ }^{3}$ Safmed Clinic, Sosnowiec, Poland

\title{
Comparison of two methods of cervical spine pain manual therapy using clinical and biochemical pain markers
}

\author{
Corresponding author: \\ Paweł Kiczmer, Department \\ of Medical and Molecular Biology, \\ 19 Jordana Str., 41-800, Zabrze, \\ e-mail: pkiczmer@wp.eu
}

Medical Research Journal 2019; Volume 4, Number 3, 163-170 10.5603/MRJ.a2019.0034 Copyright (C) 2019 Via Medica ISSN 2451-2591

\begin{abstract}
Background. Sedentary lifestyle, often associated with faulty posture is a widespread facilitating factor for cervical spine dysfunction (CSD).

Objective. The purpose of our study was to compare two methods of physical therapy for CSD: the McKenzie method and suboccipital relaxation. We investigated the effect of these methods on pain level perceived by patients and their physical fitness. The levels of biochemical stress indicators were assessed. Materials and methods. Eighty-six adult patients divided into two groups: A and B. Group A included 42 patients treated with the McKenzie method. Group B consisted of 44 patients, who underwent suboccipital relaxation. The treatment in both groups comprised 3 treatment sessions over a 6 -week period. To assess salivary slgA concentration, the ELISA technique was used. The alpha-amylase activity was determined using static method. Pain assessment was performed using the VAS scale. Disability level was evaluated with the NDI scale.

Results. Significant decrease of VAS and NDI scores were observed in both groups. An increase of slgA concentration was observed in both groups. No difference in amylase activity between the groups was observed, however, time and group effects the interaction was found to be significant. A significant correlation between both biochemical markers and VAS score was observed in group B and in the general population. Conclusions. Both therapies improve patient outcomes, however, at present we cannot indicate the advantage any method.
\end{abstract}

Key words: McKenzie method, suboccipital relaxation, neck pain, amylase; slgA

Med Res J 2019; 4 (3): 163-170

\section{Introduction}

In recent years, cervical spine dysfunction (CSD) has become a significant economic and health problem [1,2]. The development of utility electronic devices, sedentary mode of life and travelling, coupled with the lack of healthy behaviour habits are the key factors contributing to vertebral dysfunction [3]. Furthermore, early symptoms of impairment are often ignored or masked by the excessive use of easily available over-the-counter (OTC) analgesics [4]. Proper spine functioning is determined by its shape $[1,5]$, vertebral muscle tension, vertebral ligaments, joints, discs, nerves and sensory organs condition. Each of these elements plays a substantial role in maintaining somatosensory integrity of spine [6]. Impairment of any of these components may contribute towards certain types of spine dysfunction, i.e. overloading of spinal joints, which result in instability of trunk segments.

Common causes of the early stages of CSD include cervical spine kyphosis and loss of cervical lordosis. These changes of the cervical segment not only may displace the dural sac, but also affect the muscles and the ligaments of the posterior, anterior and central column. Subsequent posterior displacement of the nucleus pulposus may result in protrusion of the annulus fibrosus towards the spinal canal leading to nerve root remodelling and to increase of the pressure exerted on the nerve structures. 
The compression of the nerve roots impairs proper function both of the brachial and cervical plexuses. This leads to the manifestation of symptoms such as pain, paresthesia, muscle weakness or limitation of motion range in one or more segments. Additionally it may also cause a dysfunction of the peripheral nerves [7].

Reestablishing proper posture, as close as possible to the physiological cervical lordosis is one of the first steps of CSD treatment [5]. The restoration of proprioception in the suboccipital area is another key factor in the process of posture correction. Adequate function of proprioception receptors relies not only on suitable circulation and good muscle tone but also on patient education regarding correct posture. These therapeutic goals can be achieved using two methods - the Mechanical Diagnosis and Therapy (MDT, also called the McKenzie method) and suboccipital relaxation [8].

The idea of the McKenzie method (MDT) is to reconstruct the two basic motion patterns in the cervical spine - the retraction and hyperextension. The retraction is described as the backward head movement which creates lordosis. Hyperextension influences the displaced nucleus pulposus [9] and relaxes the posterior ligament structures, thus relieving the pressure exerted on the nerves [10]. In some patients, mentioned therapy also includes cervical spine traction.

The suboccipital relaxation is an osteopathic method, which is dedicated to improving the function of the structures of the craniospinal region, as well as the surrounding soft tissues [11]. It results in the relaxation of these structures, lowers the muscle tone and reduces blood stasis in suboccipital and perivertebral muscles [12].

Saliva constitutes a good study material in stress research. Sample collection of saliva does not generate stress related to blood vessel puncture, in contrary to venous blood sample collection. Moreover, patients are more eager to handle multiple measurements. Many factors which are considered as biomarkers of stress have been isolated from saliva. They include the secretory form of $\lg A(\operatorname{sg} A)$ which is the most prevalent antibody of the mucous membrane. It plays a crucial role in the defense against pathogens in the human body. The concentration of sigA in saliva depends on the condition of the organism. The exposure to chronic pain lowers sigA level, which corresponds to the fact of slgA being a pain biomarker $[13,14]$. The increase in slgA concentration has been reported after application of manual therapy and acupuncture $[15,16]$.

The second marker of pain we analysed is amylase - an enzyme present in the saliva, responsible for the break-down of polysaccharides. The correlation between the activity of amylase and pain sensation has been described by Shirasaki et al. [17]. Furthermore, significant association of this enzyme with stress ex- posure [18] as well as with tension headache [19] has been reported.

The purpose of our study was an attempt to apply the above-mentioned pain markers in the assessment of the two cervical spine dysfunction treatment methods: McKenzie therapy and suboccipital relaxation. To evaluate the perceived pain level and life quality we applied the VAS and NDI scales.

Hypothesis: Both examined methods affect sIgA and alpha-amylase levels in patients suffering from cervical spine pain. One method is more effective than the other.

\section{Material and methods}

The study was designed according to CONSORT statement(20). Eighty-six adult patients who met the inclusion criteria were divided into two groups, Group A and Group B. Group A included 42 patients (34 women, 8 men) treated using the McKenzie method. Group B consisted of 44 patients (33 women, 11 men) subjected to suboccipital relaxation. The average age was 50,12 years $(S D=10.40$, range $26-64)$ in the first group and 48,07 years (SD $=10.80$, range $26-64$ ) in the second group. No statistically significant differences in age or sex ratio between the two groups $(p>0.05)$ were found.

During the experiment changes of trial, design was not applied. We assumed that the sample size should include 40 patients, which is the medium number of cervical spondylosis cases in our centre per month. Due to small sample size we performed only a simple randomization method. Each patient was described with a letter of group, sequential number, number of visits, the letter "I" before, and "V" after the manipulation was done. Medical front desk receptionist generated random allocation sequence, the physio team enrolled the participants and the physicians assigned them to the interventions.

In each case, CSD was confirmed using magnetic resonance imaging (MRI). Inclusion criteria comprised: diagnosis of cervical spine pain lasting more than 4 weeks, loss or restriction of movement in the cervical spine, spondylosis and spondyloarthrosis with persistent or intermittent pain or CSD related headaches, patient age $>18$ years and written consent for participation. We excluded patients with cervical spine or head trauma, muscle weakness caused by a critical stenosis of the spinal or root canal, coexistence of carpal tunnel syndrome or ulnar nerve compression, constant pain-relieving pharmacotherapy, drug abuse, neoplastic diseases, myasthenia gravis, steroid therapy, antiobiotic or antiviral therapy, Cushing's Syndrome, Arnold-Chiari syndrome, syringomyelia, and other congenital defects of the head and the cervical area. The lack of informed 
consent was also an excluding factor. The study was approved by the Committee on the Research Ethics of the Silesian Chamber of Physicians (Komisja Bioetyczna Śląskiej Izby Lekarskiej No. 46/2015).

Each patient underwent 3 treatment sessions, one in every 3 weeks. The pain assessment was performed 6 times using the VAS scale, prior to and 20 minutes after each session. The disability level was evaluated with the NDI scale, once before each treatment session. To assess the hormone levels, saliva was collected using "salivette" type test tubes. The patients had to abstain from below mentioned activities for 20 minutes before the sample collection. The restrictions included eating, smoking cigarettes, brushing teeth, chewing gum and drinking. After collection the samples were then centrifuged and frozen at $-85^{\circ} \mathrm{C}$ for storage. The laboratory examinations were performed in the Chair and Department of Medical and Molecular Biology of the School of Medicine and Dentistry in Zabrze, Medical University of Silesia in Katowice.

\section{VAS scale}

The VAS is a reliable tool for the assessment of pain intensity. The scale is represented by a $10 \mathrm{~cm}$ coloured ruler, with its ends defining the extreme limits. The left margin represents a lack of pain, while the right one the strongest pain imaginable. Patients indicate the pain level using his finger [21, 22].

\section{NDI score}

The NDI is a questionnaire to be completed by the patient. It includes 10 questions assessing the impact of neck pain on daily life activities. The index can be used as a self-report measure of neck pain [23].

\section{Assessment of $\alpha$-amylase activity}

To assess the activity of $\alpha$-amylase we applied a static method (Aqua-Med., Łódź, Poland). This method uses 2-chloro-4-nitrophenyl-maltotrioside as substrate. The reaction was conducted in MES buffer in $\mathrm{pH}=6,0$ and at temp $=37^{\circ} \mathrm{C}$. The spectrophotometric analysis of the coloured product was performed at $405 \mathrm{~nm}$ wavelength. Saliva samples were diluted 100 times with a $0.9 \% \mathrm{NaCl}$ solution. The obtained results are presented in units of salivary $\alpha$-amylase activity $\mathrm{U} / \mathrm{ml}$.

\section{Determination of the concentration of secretory immunoglobulin class $\mathrm{A}$ (slgA)}

The ELISA kit from Immunodiagnostic AG (Germany) was used to determine sIgA concentration. The analytical procedure was performed in accordance with the instruction given by the manufacturer. Absorbance readings were made using the $\mu$ Quant reader (BioTek, USA), whereas the results were processed using the KCJunior program (BioTek, USA). The sensitivity of the method was $2.5 \mu \mathrm{g} / \mathrm{ml}$.

\section{Statistical analysis}

For statistical analysis of the obtained data, Statistica 10.0PL software was used. The average patient age was expressed as average \pm standard deviation (SD). The results of reproducible measurements were presented as average $\pm 95 \%$ confidence interval $(\mathrm{Cl})$. To compare age in both groups, t-Student test was used. For the evaluation of biochemical determinants as well as the VAS and NDI scales, ANOVA analysis of repeated measures was applied. To assess the significantly different values, we used the post hoc Scheffe test. We acknowledged parameters with a p-value $<0.05$ as statistically significant. Pearson correlation coefficient was used to evaluate correlation between variables.

\section{Results}

A significant decrease of VAS scores during the experiment was observed in both groups. Analysis of variance did not demonstrate any differences in effectiveness between the groups. However, the interaction between group and time effects was found to be significant. Patients undergoing suboccipital relaxation were characterized by greater pain recurrence compared with the McKenzie method group (Tab. 1, Fig. 1).

We observed a decrease of NDI score during the experiment. The group effect for NDI score turned out to be non-significant, as well as the interaction between time and group effects (Tab. 2).

Statistical analysis revealed a significant influence of the type of therapy on sigA levels. The time effect and the interaction between group and time effect were also found to be significant. In both groups, the final concentration of salivary slgA was higher than the starting concentration before treatment. Patients treated by suboccipital relaxation had higher final slgA concentration compared with McKenzie method patients (Tab. 3, Fig. 2).

We observed no significant group effect regarding amylase activity. However, the time effect and the interaction between group and time effect were significant. In patients undergoing McKenzie therapy, the amylase activity increased after each procedure and decreased between treatments. In patients undergoing suboccipital relaxation, we noted a significant increase in amylase concentration after first treatment and significant decrease between first and second treatment. 
Table 1. Vas scale values among patients

\begin{tabular}{lccccccccc}
\hline \multicolumn{7}{c}{ VAS } \\
\hline Group & Measurement & \multicolumn{2}{c}{$\mathbf{1}$} & \multicolumn{2}{c}{$\mathbf{2}$} & & $\mathbf{3}$ & $\mathbf{p}$ \\
\hline A & Before & 4.16 & $(3.55-4.77)$ & 3.39 & $(2.67-4.10)$ & 2.25 & $(1.51-2.99)$ & $<0.05$ \\
& After & 3.24 & $(2.58-3.89)$ & 2.59 & $(1.83-3.34)$ & 1.89 & $(1.05-2.73)$ & \\
B & Before & 4.26 & $(3.67-4.84)$ & 4.28 & $(3.57-4.99)$ & 3.11 & $(2.29-3.94)$ & $<0.05$ \\
& Afer & 2.51 & $(1.94-3.09)$ & 2.57 & $(1.98-3.17)$ & 1.90 & $(1.21-2.60)$ & \\
A vs B & $\mathrm{p}>0.05$ & & VAS* A/B & $\mathrm{p}<0.05$ & & & & \\
\hline
\end{tabular}

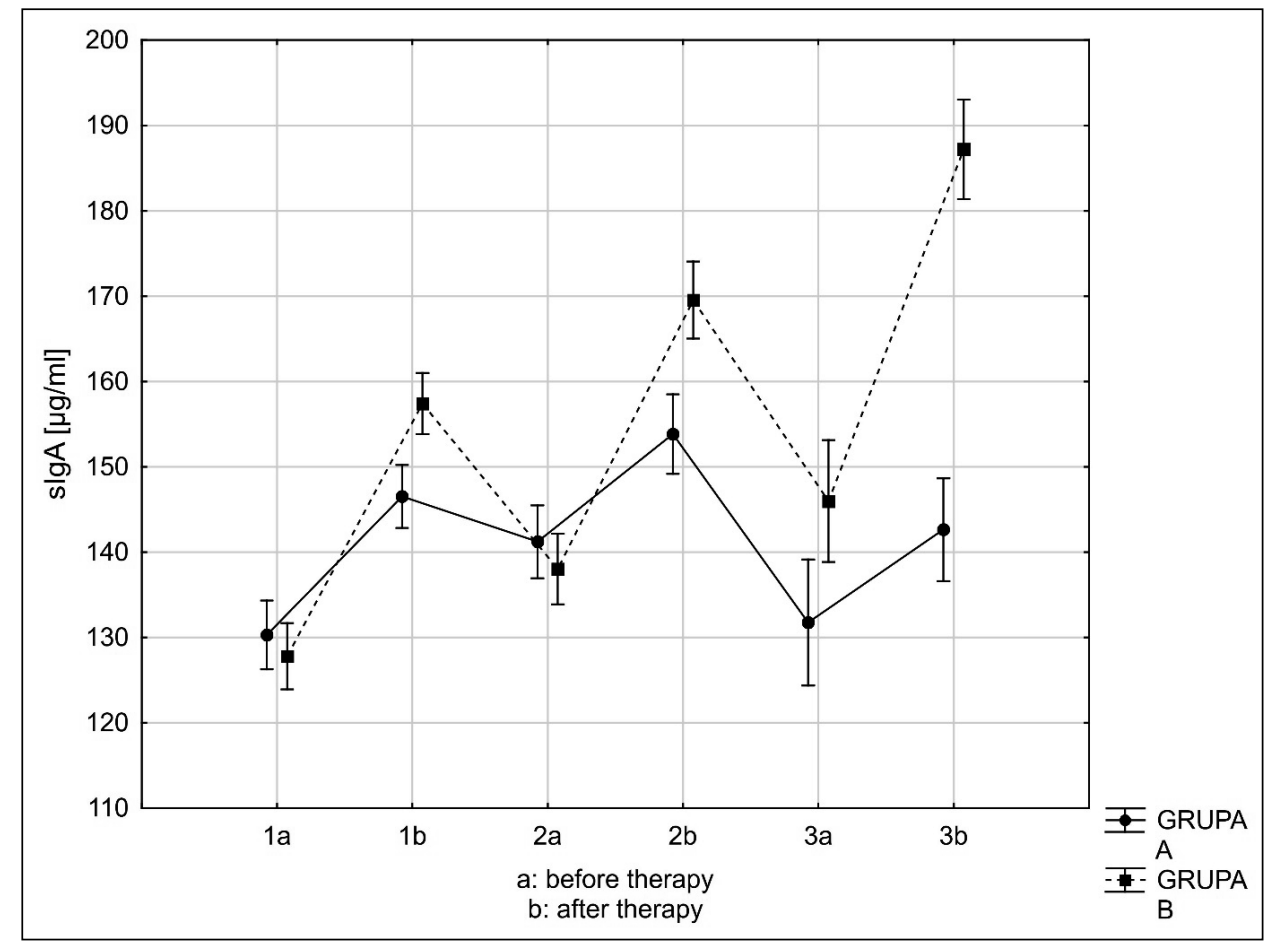

Figure 1. Perceived pain intensity in both groups expressed as VAS scores represented as mean $+/-95 \% \mathrm{Cl}$

Table 2. NDI scale among patients

\begin{tabular}{llllllll}
\hline \multicolumn{7}{c}{ Group } & \multicolumn{7}{c}{ NDI } \\
\hline A & 1 & & 2 & & 3 & & P \\
B & 13.81 & $(11.94-15.67)$ & 11.13 & $(9.39-12.87)$ & 9.87 & $(7.82-11.92)$ & $<0.05$ \\
A vs B & 14.65 & $(12.78-16.51)$ & 11.77 & $(10.04-16.51)$ & 11.29 & $(9.24-13.34)$ & $<0.05$ \\
\end{tabular}

The amylase level after second treatment remained unchanged, whereas it increased after the third intervention (Tab. 4, Fig 3).

We correlated the biochemical parameters values with VAS scores. In the analysis, we included all the results obtained during the experiment. The amylase concentration correlated negatively with VAS scores in general patient population $(R=-0.11, p=0.023)$ and in suboccipital relaxation patients $(R=-0.14$, $p=0.04)$. This association was not observed in patients undergoing McKenzie therapy $(R=-0.09$, $\mathrm{p}=0.2)$.

Regarding slgA, the correlation with VAS score was negative in the general patient population $(R=-0.19$, 
Table 3. slgA saliary concentrations among patients

\begin{tabular}{|c|c|c|c|c|c|c|c|c|}
\hline \multicolumn{9}{|c|}{$\operatorname{slgA}[\mu \mathrm{g} / \mathrm{ml}]$} \\
\hline Group & Measurement & & 1 & & 2 & & 3 & $\mathbf{p}$ \\
\hline \multirow[t]{2}{*}{$A$} & Before & 130.30 & (126.28-134.32) & 141.22 & (136.95-145.49) & 131.14 & (126.51-135.77) & $<0.05$ \\
\hline & After & 146.53 & (142.84-150.2) & 153.83 & (149.17-158.49) & 142.63 & $(136.61-148.65)$ & \\
\hline \multirow[t]{2}{*}{ B } & Before & 127.79 & $(123.90-131.69)$ & 138.01 & (133.87-142.15) & 145.96 & (136.79-155.15) & $<0.05$ \\
\hline & After & 157.42 & (153.85-160.99) & 169.53 & (165.01-174.05) & 187.21 & $(181.37-193.04)$ & \\
\hline$A v s B$ & $p<0.05$ & & $\operatorname{slg} A^{*} A / B$ & $p<0.05$ & & & & \\
\hline
\end{tabular}

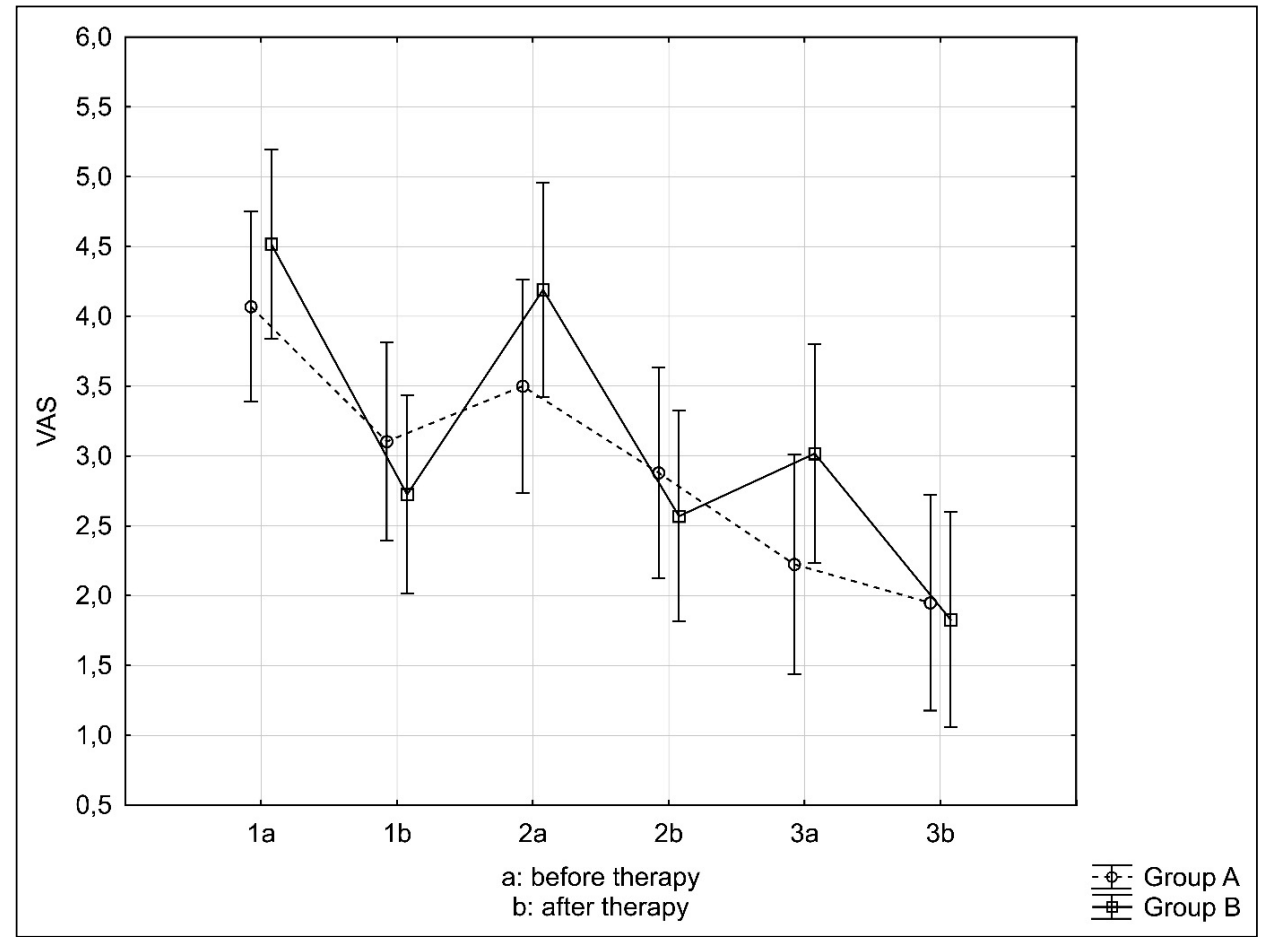

Figure 2. slgA concentrations among groups represented as mean $+/-95 \% \mathrm{Cl}$

Table 4. Amylase concentrations among patients

\begin{tabular}{|c|c|c|c|c|c|c|c|c|}
\hline \multicolumn{9}{|c|}{$\alpha$-amylase $[\mathrm{U} / \mathrm{ml}]$} \\
\hline \multirow{2}{*}{ Group } & \multirow{2}{*}{$\begin{array}{l}\text { Measurement } \\
\text { Before }\end{array}$} & \multicolumn{2}{|r|}{1} & \multicolumn{2}{|c|}{2} & \multicolumn{2}{|c|}{3} & \multirow{2}{*}{$\begin{array}{c}\mathbf{p} \\
<0.05\end{array}$} \\
\hline & & 4.64 & $(4.12-5.16)$ & 4.21 & $(3.70-4.72)$ & 3.95 & $(3.54-4.36)$ & \\
\hline & After & 5.64 & $(5.14-6.14)$ & 5.34 & $(4.82-5.86)$ & 4.75 & $(4.32-5.18)$ & \\
\hline \multirow[t]{2}{*}{ B } & Before & 5.21 & $(4.70-5.71)$ & 4.67 & $(4.17-5.16)$ & 4.71 & $(4.16-5.26)$ & $<0.05$ \\
\hline & After & 5.85 & $(5.36-6.33)$ & 4.67 & $(4.16-5.17)$ & 5.20 & $(4.78-5.61)$ & \\
\hline$A$ vs B & $p>0.05$ & $\begin{array}{c}\text { Amylase* } \\
\text { A/B }\end{array}$ & & $p<0.05$ & & & & \\
\hline
\end{tabular}

$p<0.001$ ) and in patients undergoing suboccipital relaxation $(R=-0.38, p<0.001)$. In McKenzie patients this association appeared non significant $(R=-0.01$, $\mathrm{p}=0.88$ ).

\section{Discussion}

In our manuscript, we compared two effective and life-quality improving therapies for patients with CSD. 


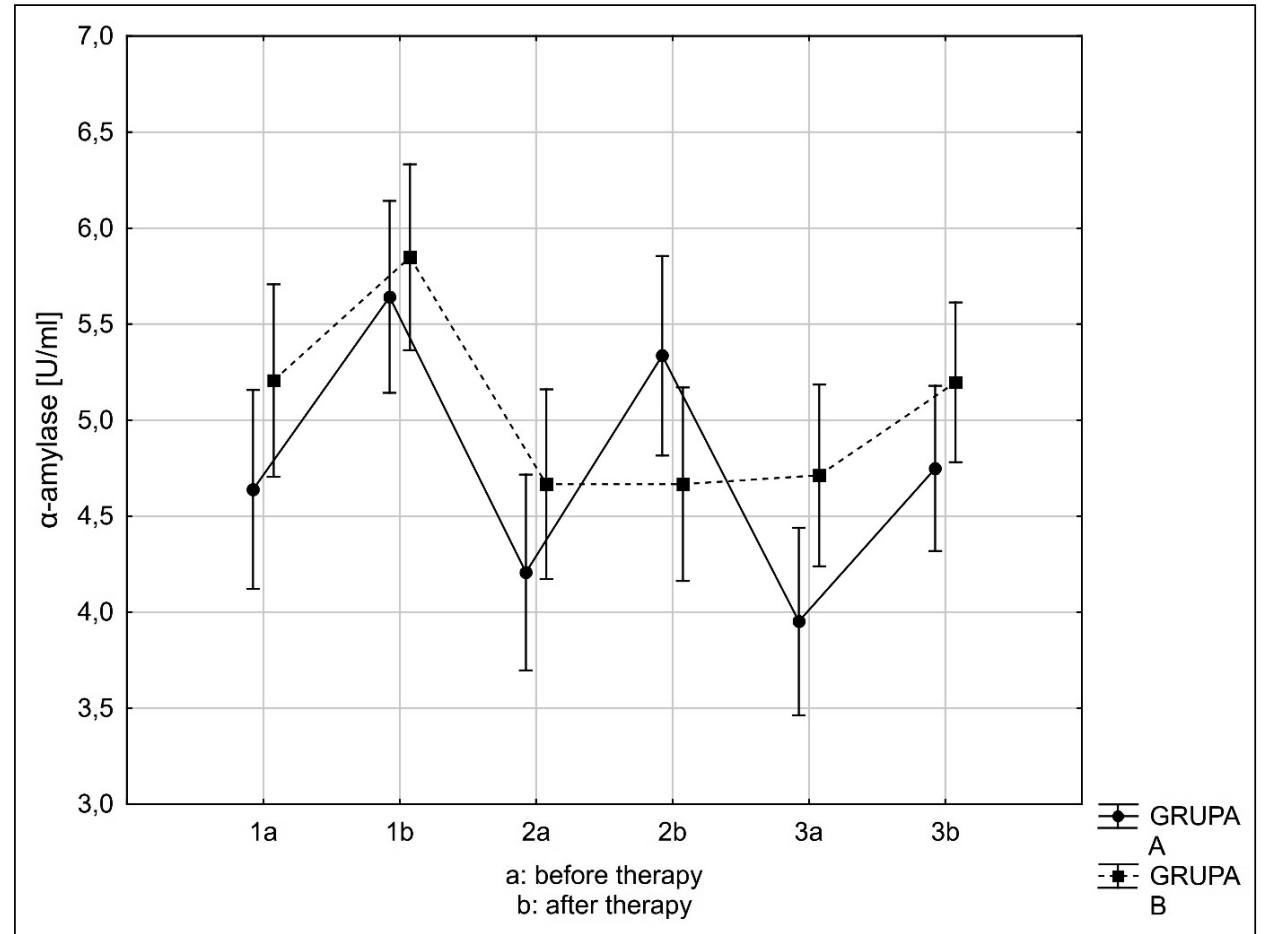

Figure 3. $\alpha$-amylase activity in subsequent measurements in both groups represented as mean $+/-95 \% \mathrm{Cl}$

The McKenzie method is an efficient tool for achieving cervical muscles relaxation, diminishing tension headaches in patients suffering from CSD [24]. Kjellman et al. reported improvement in quality of life in CSD patients undergoing this treatment method. Contrarily, Clare et al. in his systematic review did not prove unambiguous efficiency of the McKenzie method due to the lack of scientific evidence [25]. Moffet et al. described a decrease in VAS scores in patients undergoing McKenzie therapy [26]. The authors compared it to brief physiotherapy pain management. However, they did not report a significant advantage of either method. A positive influence of McKenzie therapy on patient's condition, evaluated as NDI score, was reported by Kim et al. [27].The NDI questionnaire consists of 10 categories that include activities impeded by pain in the cervical spine area. There is evidence for effectiveness of McKenzie exercise as a treatment in episodic tension-type headache resulting from cervical muscle stiffness [24].

Suboccipital relaxation therapy has also been proved effective in CSD. The effectiveness of this method has been observed in tension headache management [28, 29]. Moreover, it has been proved effective in posture disorders of the cervical spine. In our study, a significant decrease in perceived pain levels over time was observed in both groups. Aside from the pain reduction, both methods increased the quality of life of the patients which was evaluated using the NDI score. In our results, we noted a significant decrease in
NDI scores in both groups. No difference in efficiency between the two methods was observed.

Analysis of biochemical parameters allows validation of results concerning the effectiveness of evaluated methods. For this purpose, we determined the concentration of two biochemical markers of pain and chronic stress in patients' saliva.

The first evaluated marker, secretory $\lg A(\operatorname{sg} A)$, is the main immunoglobulin found in the oral cavity. Negative correlation of slgA concentration in saliva with pain intensity was described by Campos et al. [13]. According to Sobas et al. slgA may be applied as biochemical marker of pain [14]. In our study, we observed an increase of slgA level in patients undergoing suboccipital relaxation. SlgA levels were increasing significantly after each procedure and decreasing in the time between treatments.Moreover, we noted negative correlation between slgA level and VASscores in patients undergoing suboccipital relaxation. This seems to confirm the results obtained by the authors cited above.

In patients undergoing McKenzie therapy, we observed a slight increase of slgA concentration after treatment, however, the final concentration did not reach significance as compared to the initial level. Furthermore, there was no correlation between slgA level and VAS scores. The increase of slgA after manual therapy was described previously by Donoyama et al. Patients undergoing acupuncture treatment demonstrated higher slgA level which was also associated with pain reduction [16]. The 
significant increase in slgA concentration and a strong negative correlation with VAS scores in patients undergoing suboccipital relaxation may indicate better effectiveness of this method in comparison to McKenzie therapy. These results need to be confirmed by a study comprising greater sample size and longer examination period.

The second investigated biomarker was $\alpha$-amylase. Its positive correlation with pain intensity was described by Shirasaki et al. [17]. A significant decrease in amylase activity was noted in patients suffering from neck pain, who underwent chiropractic treatment [30].

In our study, patients undergoing McKenzie therapy were characterized by significant changes in amylase activity in saliva. The activity increased after each procedure and thereafter decreased reaching the initial value between the procedures. Suboccipital relaxation therapy did not influence on amylase level significantly. Moreover, we noted a negative correlation between amylase level and VAS scores.

The results indicate that suboccipital relaxation method may be associated with less pain (caused by the treatment itself) compared with McKenzie therapy.

Both evaluated methods led to the reduction of pain in patients. The VAS and NDI scale analysis did not show advantage of one method over another. However, the results of biochemical markers evaluation speak for suboccipital relaxation method. In this group of patients, we observed an increase in slgA level, which is negatively correlated with pain sensation. The evaluation of amylase levels showed more beneficial effect of this method on patients' condition.

Our study is limited by the number of patients in both groups.It should be taken into account that, to the best of our knowledge, this is the first study analysing biochemical markers of pain in McKenzie and suboccipital relaxation methods. It allows validation of the obtained results and further comparison with the subjective feeling of the patient. Both indicators showed a significant correlation with the VAS scale in patients undergoing suboccipital relaxation. This association was not observed in patients treated with McKenzie therapy. We believe that the significant association between individual feeling of the patient (expressed as VAS scale score) with biochemical parameters may indicate the effectiveness of the given therapy. In the future, we are planning to conduct a similar study with greater sample size and longer examination time, which may improve efficiency evaluation of both treatment methods.

\section{Conclusion}

Our results suggest that both therapies improve patients outcomes, as represented by the VAS and NDI scores, however, the examined biochemical markers indicate the advantage of suboccipital relaxation over McKenzie method. Our study is the first examination comparing the McKenzie method with the suboccipital relaxation and correlating them with slgA and amylase saliva levels. This pilot research may give grounds for further research evaluating the use of the suboccipital relaxation and the McKenzie method in cervical spine disorders.

\section{Abbreviations}

CSD - cervical spine dysfunction, VAS - visual analogue pain scale, NDI — neck disability index, MDT - McKenzie method

\section{Conflict of Interests}

None declared.

\section{References}

1. Tatu L, Jost WH. Anatomy and cervical dystonia : "Dysfunction follows form". J Neural Transm (Vienna). 2017; 124(2): 237-243, doi: 10.1007/s00702-016-1621-7, indexed in Pubmed: 27624726.

2. Thompson DP, Woby SR. Acceptance in chronic neck pain: associations with disability and fear avoidance beliefs. Int J Rehabil Res. 2017; 40(3): 220-226, doi: 10.1097/MRR.0000000000000230, indexed in Pubmed: 28445326 .

3. Cohen S. Epidemiology, Diagnosis, and Treatment of Neck Pain Mayo Clinic Proceedings. 2015; 90(2): 284-299, doi: 10.1016/j mayocp.2014.09.008.

4. Ferrara LA. The biomechanics of cervical spondylosis. Adv Orthop. 2012; 2012: 493605, doi: 10.1155/2012/493605, indexed in Pubmed: 22400120

5. Lippa L, Lippa L, Cacciola F. Loss of cervical lordosis: What is the prognosis? J Craniovertebr Junction Spine. 2017; 8(1): 9-14, doi: 10.4103/0974-8237.199877, indexed in Pubmed: 28250631

6. Cramer GD, Darby SA. Basic and clinical anatomy of the spine, spinal cord, and ANS. 2nd ed. St. Louis: Elsevier Mosby; 2005. 1 online resource $(x x . ; 652$.

7. Benzel EC. Spine surgery: Techniques, complication avoidance, and management. 2nd ed. New York: Churchill Livingstone; . XXIX Seiten, Seite 1131. 2205; 31: Seiten.

8. Clare HA, Adams R, Maher CG. A systematic review of efficacy of McKenzie therapy for spinal pain. Aust J Physiother. 2004; 50 (4): 209-216, indexed in Pubmed: 15574109.

9. Werneke M, Hart DL, Cook D. A descriptive study of the centralization phenomenon. A prospective analysis. Spine (Phila Pa 1976). 1999 24(7): 676-683, doi: 10.1097/00007632-199904010-00012, indexed in Pubmed: 10209797.

10. Mercer S, Bogduk N. The ligaments and annulus fibrosus of human adult cervical intervertebral discs. Spine (Phila Pa 1976). 1999; 24(7): 619-26; discussion 627, doi: 10.1097/00007632-19990401000002, indexed in Pubmed: 10209789.

11. Franke H, Franke JD, Fryer G. Osteopathic manipulative treatment for nonspecific low back pain: a systematic review and meta-analysis. BMC Musculoskelet Disord. 2014; 15: 286, doi: 10.1186/14712474-15-286, indexed in Pubmed: 25175885.

12. Haller H, Lauche $\mathrm{R}$, Cramer $\mathrm{H}$, et al. Craniosacral Therapy for the Treatment of Chronic Neck Pain: A Randomized Sham-controlled Trial. Clin J Pain. 2016; 32(5): 441-449, doi: 10.1097/AJP.0000000000000290, indexed in Pubmed: 26340656.

13. da Silva Campos MJ, Souza Alves CC, Barbosa Raposo NR, et al. Influence of salivary secretory immunoglobulin A level on the pain experienced by orthodontic patients. Med Sci Monit. 2010; 16(9): CR405-CR409, indexed in Pubmed: 20802411. 
14. Sobas EM, Reinoso R, Cuadrado-Asensio R, et al. Reliability of Potential Pain Biomarkers in the Saliva of Healthy Subjects: Inter-Individual Differences and Intersession Variability. PLoS One. 2016; 11(12) e0166976, doi: 10.1371/journal.pone.0166976, indexed in Pubmed 27907037

15. Donoyama N, Shibasaki M. Differences in practitioners' proficiency affect the effectiveness of massage therapy on physical and psychological states. J Bodyw Mov Ther. 2010; 14(3): 239-244, doi: 10.1016/j. jbmt.2009.01.007, indexed in Pubmed: 20538221.

16. Matsubara Y, Shimizu K, Tanimura Y, et al. Effect of acupuncture on salivary immunoglobulin A after a bout of intense exercise. Acupunct Med. 2010; 28(1): 28-32, doi: 10.1136/aim.2009.001677, indexed in Pubmed: 20351374.

17. Shirasaki S, Fujii H, Takahashi M, et al. Correlation between salivary alpha-amylase activity and pain scale in patients with chronic pain. Reg Anesth Pain Med. 2007; 32(2): 120-123, doi: 10.1016/j rapm.2006.11.008, indexed in Pubmed: 17350522.

18. Takai N, Yamaguchi M, Aragaki T, et al. Effect of psychological stress on the salivary cortisol and amylase levels in healthy young adults. Arch Oral Biol. 2004; 49(12): 963-968, doi: 10.1016/j.archoralbio 2004.06.007, indexed in Pubmed: 15485637.

19. Vahedi M, Mazdeh M, Hajilooi M, et al. The Relationship Between Salivary Alpha Amylase Activity and Score of McGill Pain Questionnaire in Patients With Tension Type Headache. Basic Clin Neurosci. 2018; 9(1) 59-64, doi: 10.29252/nirp.bcn.9.1.59, indexed in Pubmed: 29942441

20. Schulz KF, Altman DG, Moher D, et al. CONSORT Group. CONSORT 2010 Statement: updated guidelines for reporting parallel group randomised trials. BMC Med. 2010; 8: 18, doi: 10.1186/1741-7015-8-18, indexed in Pubmed: 20334633.

21. Faiz KW. [VAS--visual analog scale]. Tidsskr Nor Laegeforen. 2014; 134(3): 323, doi: 10.4045/tidsskr.13.1145, indexed in Pubmed 24518484

22. Heller GZ, Manuguerra M, Chow R. How to analyze the Visual Analogue Scale: Myths, truths and clinical relevance. Scand J Pain. 2016;
13: 67-75, doi: 10.1016/j.sjpain.2016.06.012, indexed in Pubmed: 28850536

23. MacDermid JC, Walton DM, Avery S, et al. Measurement properties of the neck disability index: a systematic review. J Orthop Sports Phys Ther. 2009; 39(5): 400-417, doi: 10.2519/jospt.2009.2930, indexed in Pubmed: 19521015

24. Choi SY, Choi JH. The effects of cervical traction, cranial rhythmic impulse, and Mckenzie exercise on headache and cervical muscle stiffness in episodic tension-type headache patients. J Phys Ther Sci. 2016; 28(3): 837-843, doi: 10.1589/jpts.28.837, indexed in Pubmed: 27134368.

25. Kjellman G, Oberg B. A randomized clinical trial comparing general exercise, McKenzie treatment and a control group in patients with neck pain. J Rehabil Med. 2002; 34(4): 183-190, indexed in Pubmed: 12201614.

26. Moffett JK, Jackson DA, Gardiner ED, et al. Randomized trial of two physiotherapy interventions for primary care neck and back pain patients: 'McKenzie' vs brief physiotherapy pain management. Rheumatology (Oxford). 2006; 45(12): 1514-1521, doi: 10.1093/rheumatology/kel339, indexed in Pubmed: 17062645.

27. Kim J, Kim S, Shim J, et al. Effects of McKenzie exercise, Kinesio taping, and myofascial release on the forward head posture. J Phys Ther Sci. 2018; 30(8): 1103-1107, doi: 10.1589/jpts.30.1103, indexed in Pubmed: 30154609.

28. Espí-López GV Arnal-Gómez A Arbós-Berenguer T, et al Effectiveness of Physical Therapy in Patients with Tension-type Headache: Literature Review. J Jpn Phys Ther Assoc. 2014; 17(1): 31-38, doi: 10.1298/jjpta.17.31, indexed in Pubmed: 25792906.

29. Yang DJ, Kang DaH. Comparison of muscular fatigue and tone of neck according to craniocervical flexion exercise and suboccipital relaxation in cervicogenic headache patients. J Phys Ther Sci. 2017; 29(5): 869-873, doi: 10.1589/jpts.29.869, indexed in Pubmed: 28603362.

30. Ogura T, Tashiro M, Masud M, et al. Cerebral metabolic changes in men after chiropractic spinal manipulation for neck pain. Altern Ther Health Med. 2011; 17(6): 12-17, indexed in Pubmed: 22314714. 